\title{
Leveraging Snow Plow Dashboards Cams and Connected Vehicle Speed Data to Improve Winter Operations Performance Measures
}

\author{
Howell Li \\ Purdue University \\ 550 Stadium Mall Drive \\ West Lafayette, IN 47906 \\ (765) 494-9601 \\ howell-li@purdue.edu \\ Logan Peters \\ Iowa State University \\ Christina Banuelos \\ Iowa State University \\ Jonathan Zaugg \\ Iowa State University \\ Anuj Sharma \\ Iowa State University \\ Darcy M. Bullock \\ Purdue University
}




\begin{abstract}
Recent developments in mobile phone applications for polling traffic and roadway condition data from the public have led to new data sources that complement traditional Intelligent Transportation System (ITS) sensors, weather stations, and automatic vehicle location (AVL) on fleet vehicles. This study compared qualitative assessments by different users of snow plow dashboard images with interstate connected vehicle speeds. The data set was composed of 1,119 geotagged images collected during a snowstorm on February 2-3, 2016 on Interstate 80. The images were classified by three users into one of seven categories to describe the road conditions. The classification of images saw the greatest deviation when road conditions were "wet," "dry," or "icy," suggesting some variation in the interpretation of these categories by the users. Connected vehicle speed data was used to validate conditions and analyze speeds at GPS locations of each image for a two-hour window around when the image was taken to identify any potential impacts the plows have made. The Kolmogorov-Smirnov test was used for all before and after combinations to determine which comparisons were the most significant between minutes before and after the plow, and found that speed data taken between 17 to 12 minutes before the plow had the greatest maximum distance improvement on the speed distributions 25 to 35 minutes after the plow has gone through. Longer term, these techniques will provide a framework for agencies to dynamically monitor changing road conditions and respond to changing weather conditions.
\end{abstract}

Keywords: Winter weather, automatic vehicle location, connected vehicle, weather imagery, winter maintenance. 


\section{INTRODUCTION}

US Federal Highway Administration's Road Weather Management Program (1) reports that around $70 \%$ of the US roadways lie in a region that has 5 or more inches of snow fall annually, and around the same percent of US population live in these regions. A quarter of all crashes are weather-related resulting in over 1,300 fatalities and 116,800 injuries every year (1). Inclement weather reduces pavement friction thus affecting safe driving speeds and vehicle maneuverability, resulting in higher crash risks. Increased crash rates and the associated secondary crash rates are a significant concern during winter weather conditions $(1,3,4)$. Pisano et. al. estimated the annual cost of weather-related crashes to range between $\$ 22$ to $\$ 51$ billion dollars. Several studies report that the risk of crash is significantly higher during the initial snow events of the season suggesting that drivers can get more acclimatized over a period of time to better maneuver the inclement weather conditions $(6,7)$. Several DOTs have started programs to provide real-time information to the drivers. As an example, Utah DOT provides road weather information using Closed-Circuit Television (CCTV) cameras and classifying the existing road conditions (8). Similarly, Iowa DOT's "Track a Plow" program uses cameras mounted on its snow plows to post real-time pictures on its user information website (9).

\subsection{The Connected Snow Plow}

Agencies across the country have adopted the use of automatic vehicle location (AVL) systems installed in their vehicle fleets. Depending on the outfitting, onboard telematics and Global Positioning System (GPS), temperature sensors, and cameras, various data are streamed real-time or archived for after-action assessment. This technology installed on snow plows can be leveraged to evaluate the conditions of the roadway as the plow travels on its designated route during a winter event. Moreover, the positions of the plows at any instant can be combined with external data sources, such as stationary Road Weather Information Systems (RWIS), Intelligent Transportation Systems (ITS) sensors, and connected vehicle data to provide a holistic assessment of the actual driver experience before and after the snow plow has gone through. 


\subsection{Crowd-Sourcing of Road Condition Assessment}

In addition to agency-generated AVL data, Utah DOT has developed a mobile phone application for public use to collect crowd-sourced roadway conditions based on user-assessed, qualitative input. Figure 2 shows screenshots of the assessment application. Figure 2a shows the selection menu for the location and the conditions of the road and weather with optional comments for input. Figure $2 b$ shows the choices a user can select from to describe the weather conditions they are currently experiencing. This study looks to evaluate the qualitative assessment of road conditions by different users and compare the results to measured speed data.

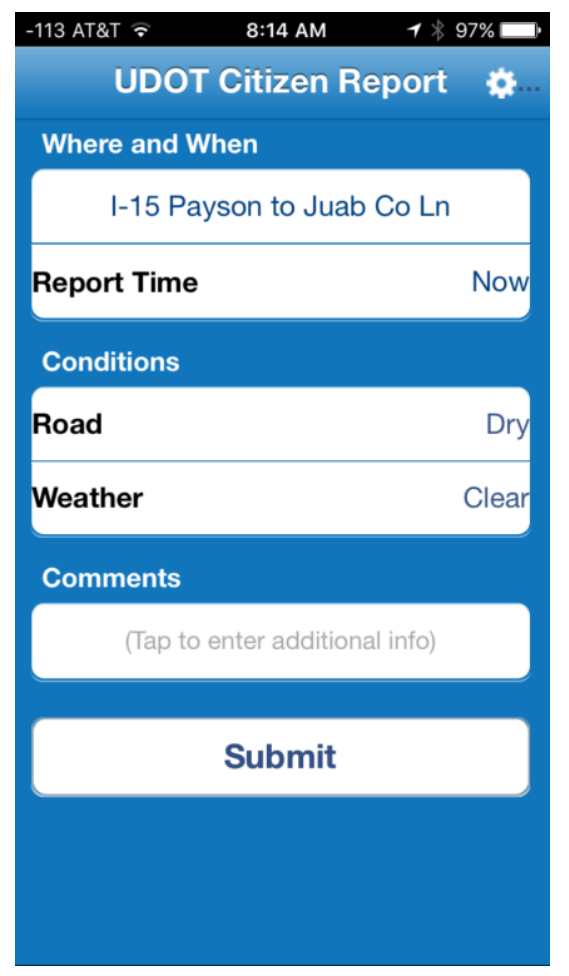

a.

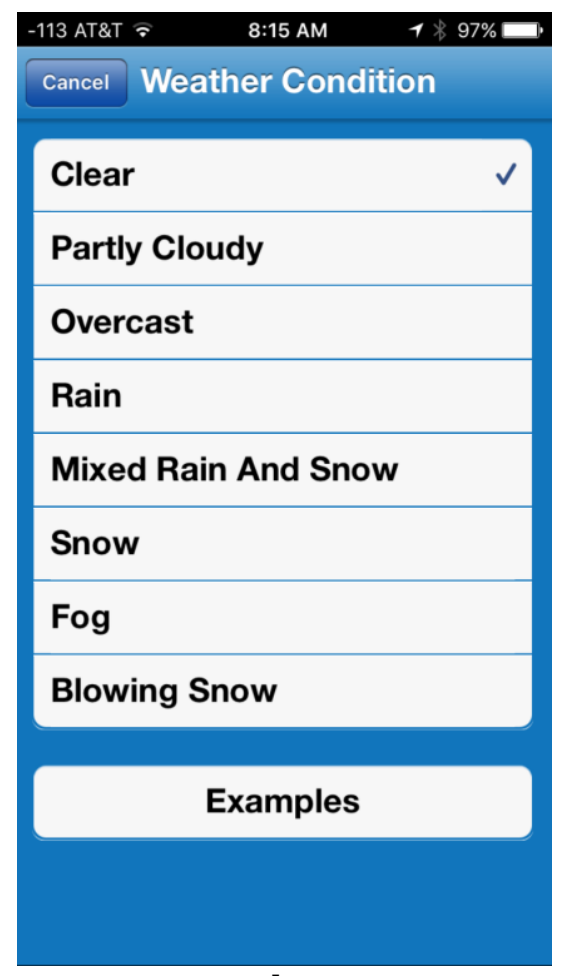

b.

Figure 1. Utah DOT Citizen Report Application

\section{METHODOLOGY}

\subsection{Assessment of Imagery}

To evaluate how effectively a user assesses road conditions, images from snow plows were collected and classified after the storm. The images were captured and geotagged at 20 minute intervals on Iowa DOT snow plows. The classifications were later paired with connected vehicle speed data for further analysis.

The image data used is for the February 2-3, 2016, snow storm on Interstate 80 in Iowa. Figure 2 shows the geo-referenced locations of 1,119 images from 29 trucks during the operation. The pictures were downloaded and categorized based on location and time. The images were then automatically sorted based on time, day or night. Finally the day images were manually categorized. 
The images were classified first by using a Java program to separate the images based on the time of day: whether the image is "light" or "dark." The program utilized red-green-blue (RGB) detection logic to distinguish dark and light pixels in each image. All nighttime photos were eliminated to mitigate any potential variation in lighting conditions. Then, the "day light" images were separated into seven categories: snow-covered, patchy snow, icy, slushy, wet, dry, and dark. Examples of images in each category is shown in Figure 3 with the exception of "dark."

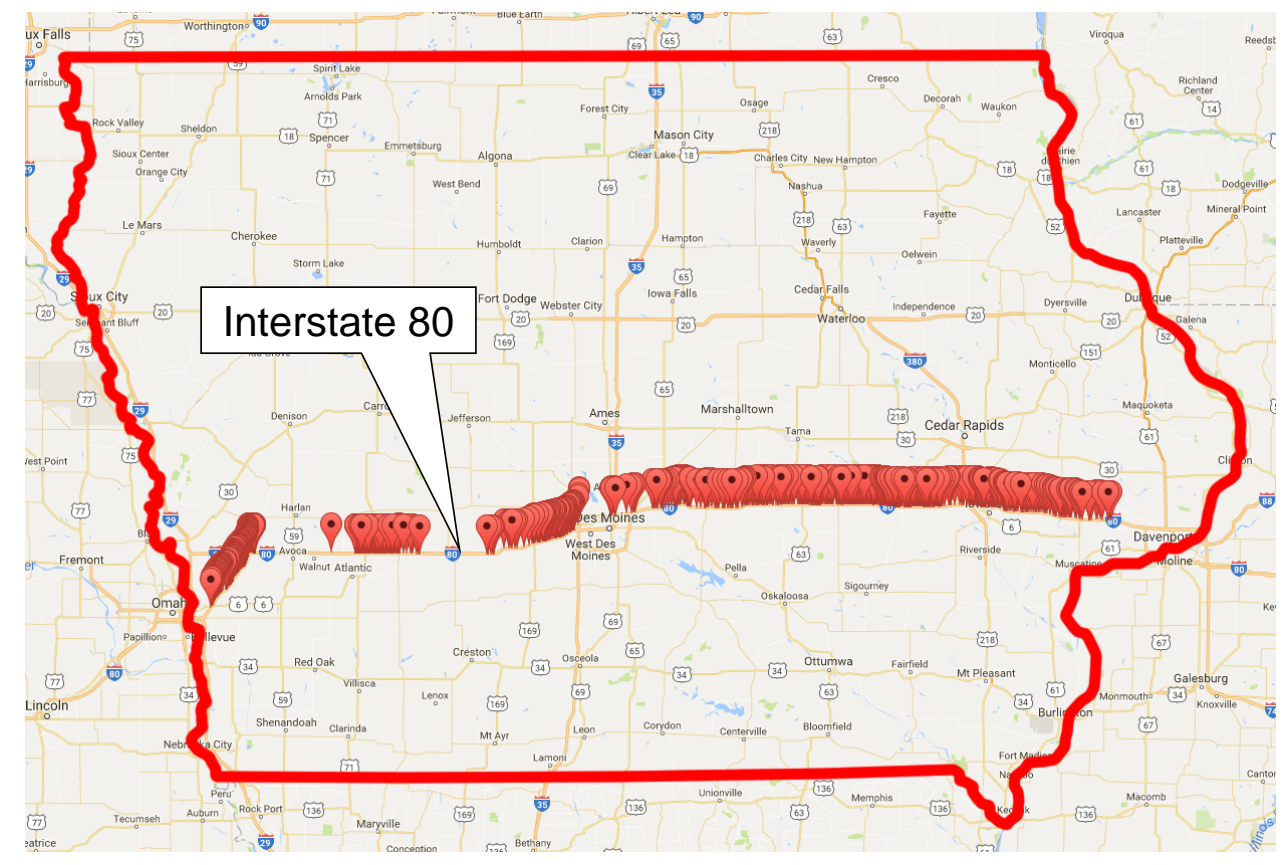

Figure 2. Locations of images captured, I-80 in Iowa (1,119 images).

An application written in Java was used to facilitate the sorting of images by multiple individuals. The application displayed the images and a number of buttons, one for each weather option. The images were then compiled into appropriate folders and an output of a commaseparated variable (CSV) file detailing the name, location, camera and weather conditions was produced.

Three users from Iowa State University then classified the same images using the six criteria in Figure 3. In total, 484 out of the 1119 images were classified as "dark" and were excluded from the evaluation. For the remaining images, 25 to 75 images were classified as "patchy snow," 83 to 151 images were classified as "icy," 54 to 84 images were classified as "slushy," 232 to 400 images were classified as "wet," and 13 to 13 to 86 images were classified as "dry." There were no images classified as "snow covered." Table 1 shows the summary of classifications by user. 


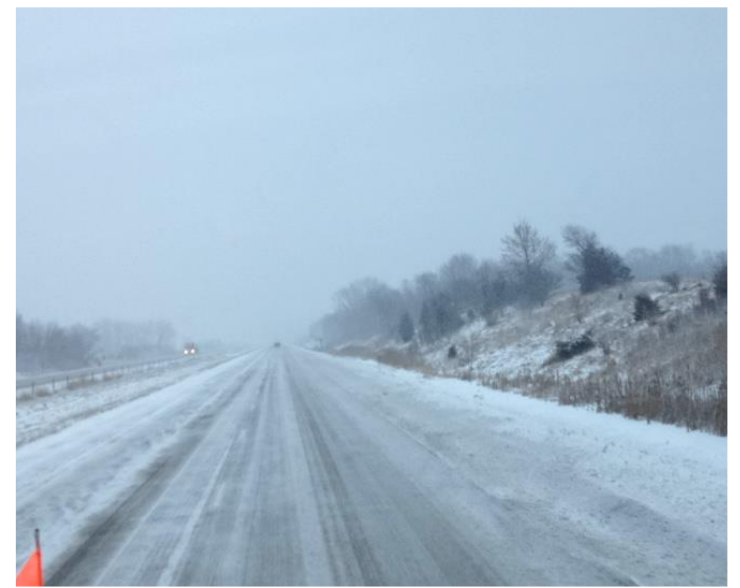

Snow Covered

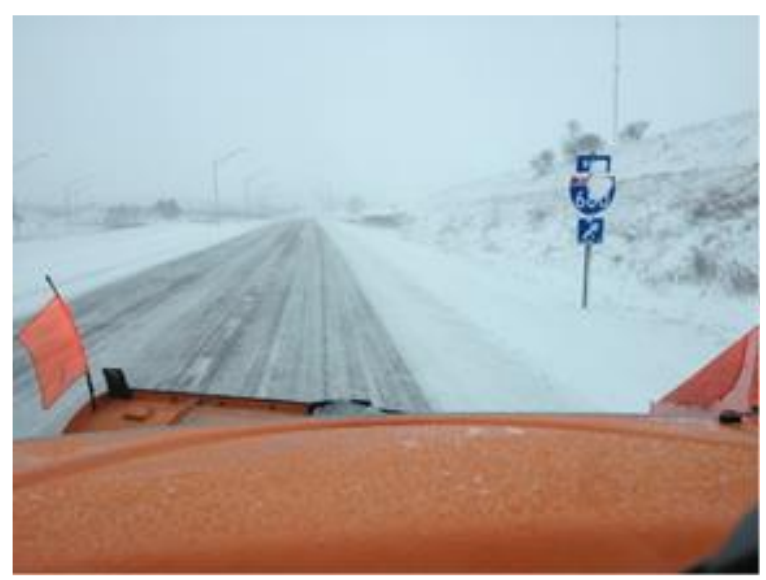

Icy

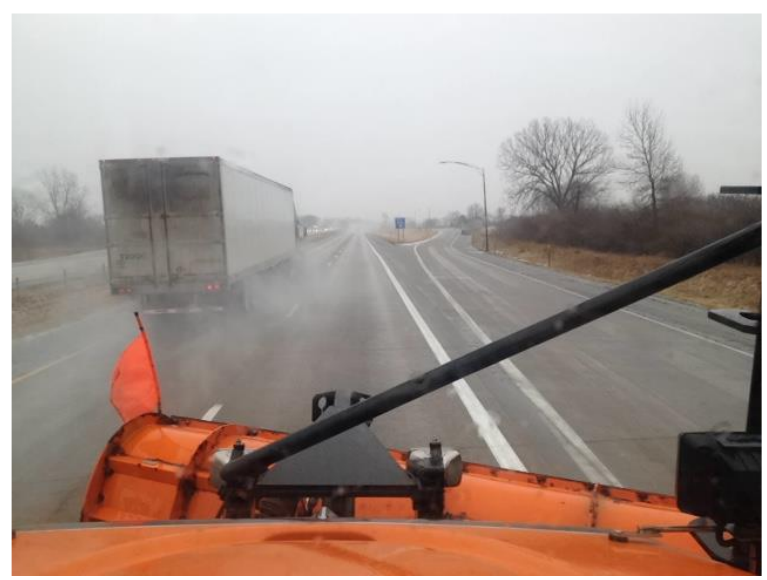

Wet

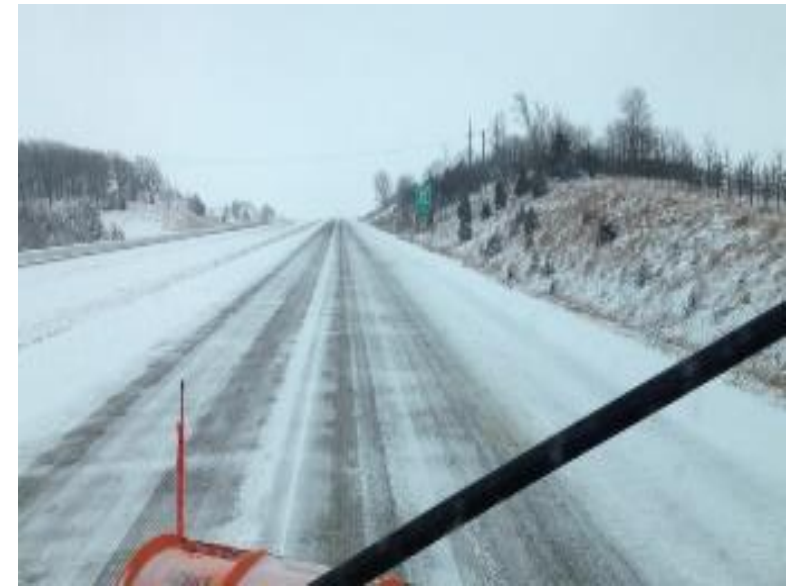

Patchy Snow

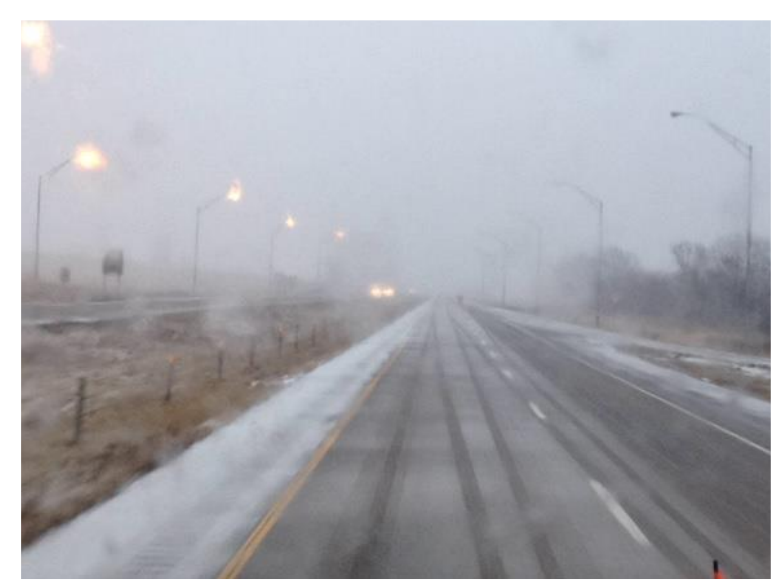

Slushy

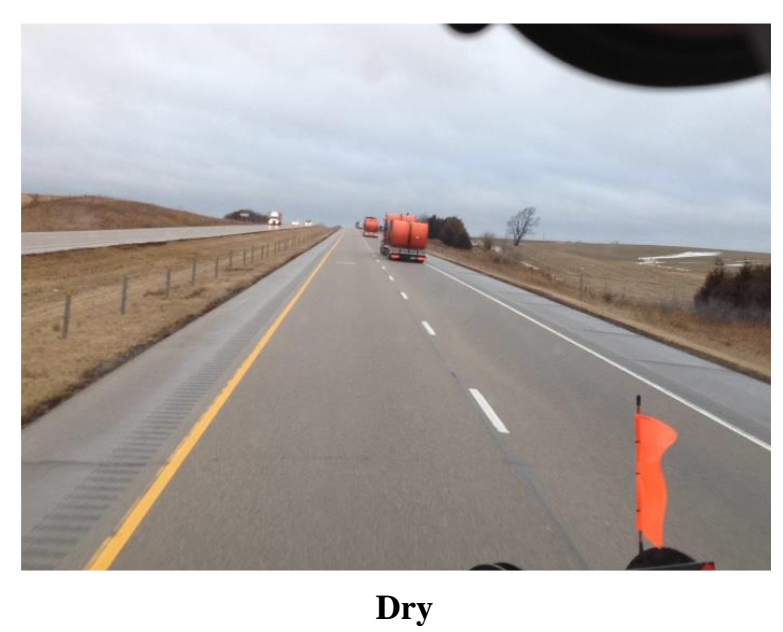

Figure 3. Different classifications of roadway conditions. 
Table 1. Summary of classified images for each user.

$\begin{array}{lcccccc} & \text { Patchy Snow } & \text { Icy } & \text { Slushy } & \text { Wet } & \text { Dry } & \text { Snow Covered } \\ \text { User 1 } & 25 & 140 & 54 & 380 & 28 & 0 \\ \text { User 2 } & 67 & 151 & 84 & 232 & 86 & 0 \\ \text { User 3 } & 75 & 83 & 64 & 400 & 13 & 0\end{array}$

\subsection{Tracking Roadway Speeds near the Plow}

To quantitatively assess the roadway conditions suggested by the image classifications, connected vehicle data along Interstate 80 in Iowa at the locations of the snow plow images (Figure 2) were collected over the duration of the storm. These data are downloaded in real-time through a traffic web service and are one-minute representative speeds on segments of roadway defined roughly one mile in length. These data originate from GPS-enabled embedded devices on commercial fleet vehicles and trucks. These speeds may also contain some lag in the response to road conditions.

For each image, geographic information system (GIS) software is used to identify the closest

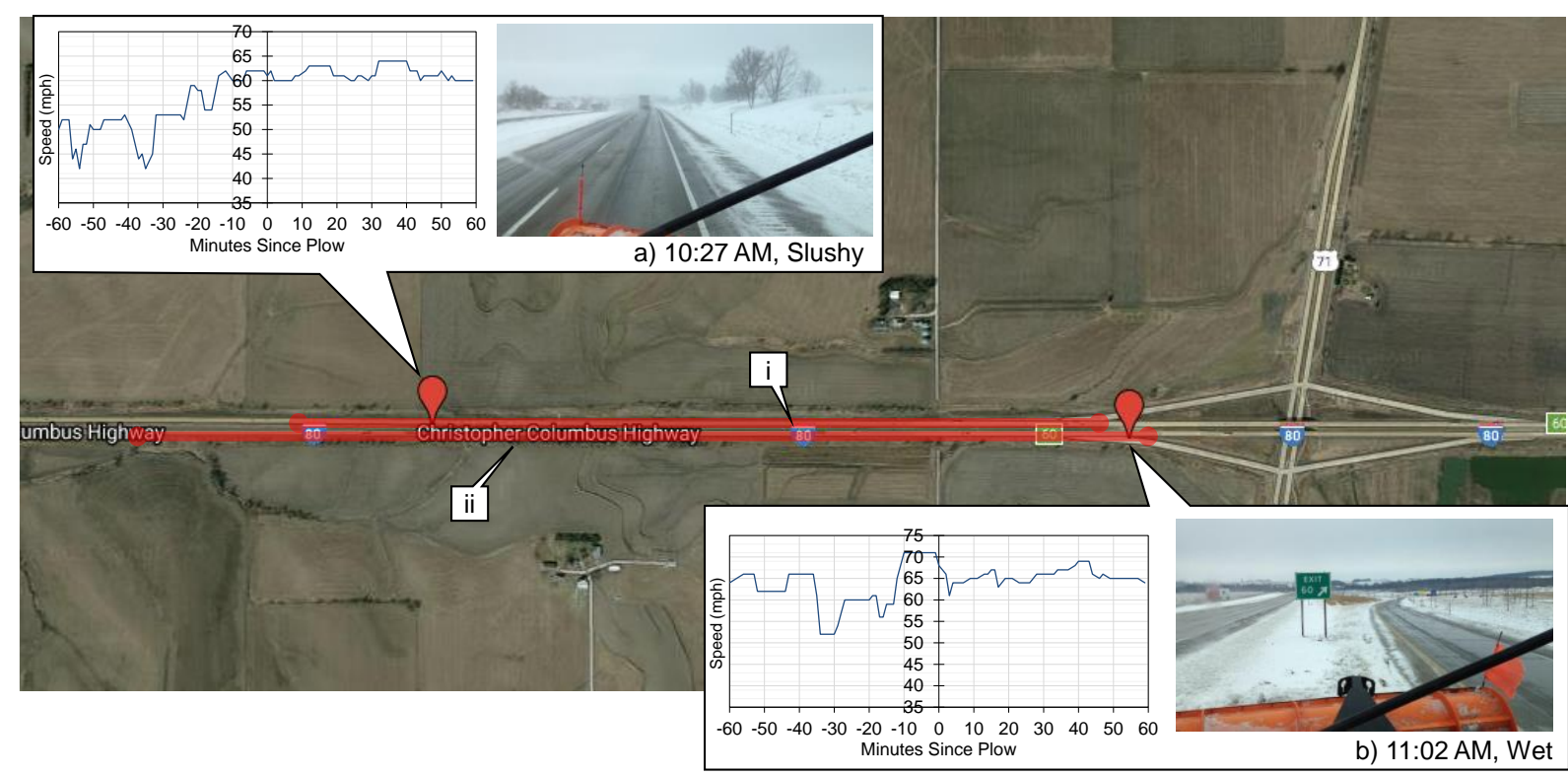

Figure 4. Speed profile of segment before and after captured plow image, February 2, 2016.

roadway segment to the latitude and longitude signature provided by the image. If a match is found, speed data for a two-hour connected vehicle data window is retrieved for the segment at the location and time of the plow's signature to characterize the performance of traffic. In Figure 4, a map is shown with two markers of where two separate images were taken on February 2, separated by 35 minutes. The two segments associated with the images are shown by callout $i$ and $i$ for the westbound and eastbound direction, respectively. The westbound segment has a length of 1.1 miles and the eastbound segment has a length of 1.3 miles. The image along with the profile of speeds one hour before and after the plow is also called out at each marker. At 10:27AM (callout $a$ ), an image of the conditions in the westbound direction just west of exit 60 was classified as "slushy." The speed profile of the westbound segment covering the image location shows that at 60 minutes to 16 minutes before the plow, the segment operated well below typical interstate speeds, anywhere 
from $59 \mathrm{MPH}$ to as low as $42 \mathrm{MPH}$ at 35 minutes before the plow's arrival. At 14 minutes before the plow's arrival, the speed on the segment improves to $61 \mathrm{MPH}$, where for the next hour the speeds remains between 60 and $64 \mathrm{MPH}$. This improvement strongly suggests the plow having a substantial impact to improve speeds during inclement conditions. The premature speed improvement before the actual recorded arrival of the plow may be attributable to the length of the segment of where the speeds are sampled relative to the plow's location, and to a delay component of data reporting.

At 11:02AM (callout $b$ ), the same plow returns in the eastbound direction exiting the interstate at exit 60 . The roadway condition at this time was now assessed as "wet." For the associated segment in the eastbound direction, the speeds are generally higher but still has a 14 MPH variation, between $66 \mathrm{MPH}$ at 53 minutes before the plow and $52 \mathrm{MPH}$ at 34 minutes before the plow. The segment improves to $71 \mathrm{MPH}$ just 10 minutes before the image was taken, after which the speeds drop off slightly. For the next hour, the segment stays between $66 \mathrm{MPH}$ and 61 MPH and has substantially less variation than the previous hour before the plow. Again, this is indicative of the plow's impact on traffic speed, and in particular, the variation in that speed.

\section{CALCULATION}

\subsection{Image Classifications over Time}

A cumulative frequency diagram that tracks the number of images classified by each user is plotted for each category over time. Figure 5a-e shows the number of images classified shown on the yaxis over the 42-hour period during the February 2-3 storm. The time is shown on the x-axis. Each figure counts the number of images for "patchy snow," "icy," "slushy," "wet," and "dry." The scale on the y-axis has been adjusted higher to show detail in the "icy" and "wet" categories. The overnight hours were not classified as most of the images were "dark," and are shown as flat lines in each graph. There were no images classified as "snow covered," and the category has been excluded here.

In Figure 5a, the "patchy snow" category has the greatest increase from the hours of 7AM to 10AM on February 2 and 7AM to 12PM on February 3. User 3 has the greatest number of classifications in this category with 75 images over the period, while User 1 has the fewest at 25 images. Comparing User 2 and User 3, the morning hours of February 2 saw over 40 classifications by User 3 while under 30 for User 2. On the morning of February 3, User 2 catches up with 38 classifications while User 3 has 32. These curves suggest there was snow on the ground on both mornings, but conditions improved by late-morning possibly due to snow plow activity.

Figure $5 \mathrm{~b}$ shows accumulations of the "icy" classification. In this category, User 2 has the greatest number of classifications at 151 images over the 42 hours, with User 1 coming close at 140 images. Callout $i$ indicates a point in time where User 1 has the most number of "icy" classifications, just before 9AM on February 2. This suggests that User 1 has classified many of what the other users considered to be "patchy snow" images into this category. Conversely, User 3 shows the fewest number of classifications in the "icy" category.

Figure 5c shows User 2 having the most number of classifications for "slushy" at 84 images, mostly accumulated on the morning of February 2. This suggests User 2 classified some of the images considered to be "patchy snow" by the other users into this category. User 1 and User 3 track pretty closely for the majority of this classification, however, callout $i i$ shows a bend in the curve for User 3 during the morning of February 3. This suggests that User 3 has classified some images of what User 1 considered as "patchy snow" into this category. 


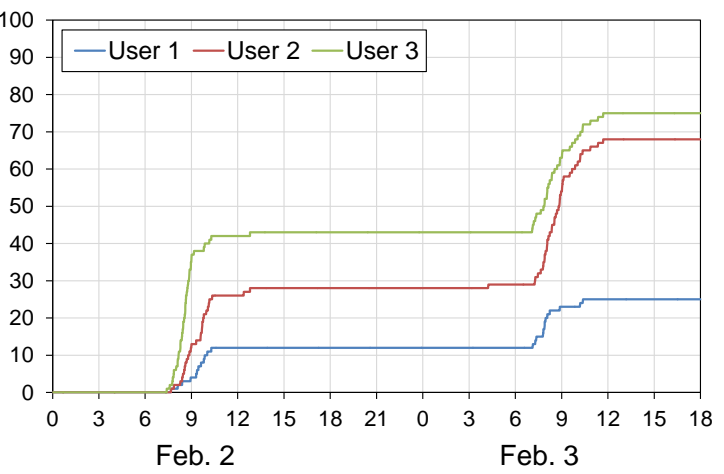

a) Patchy Snow

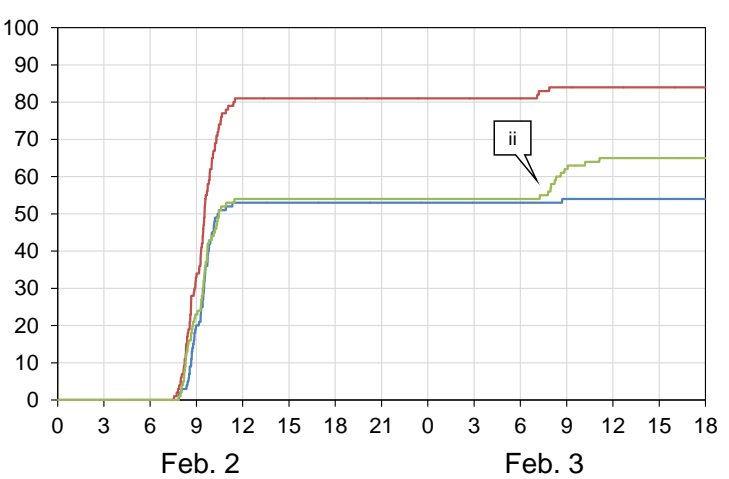

c) Slushy

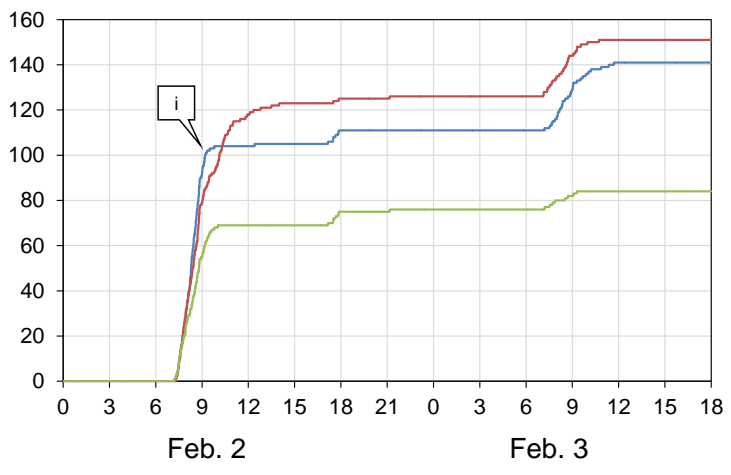

b) Icy

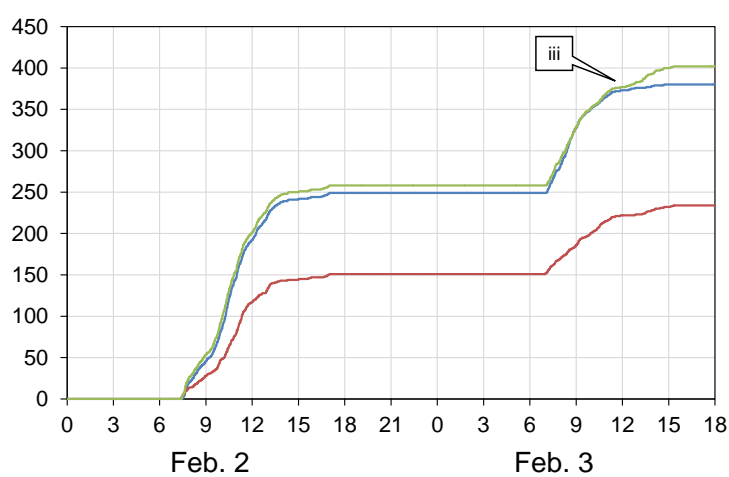

d) Wet

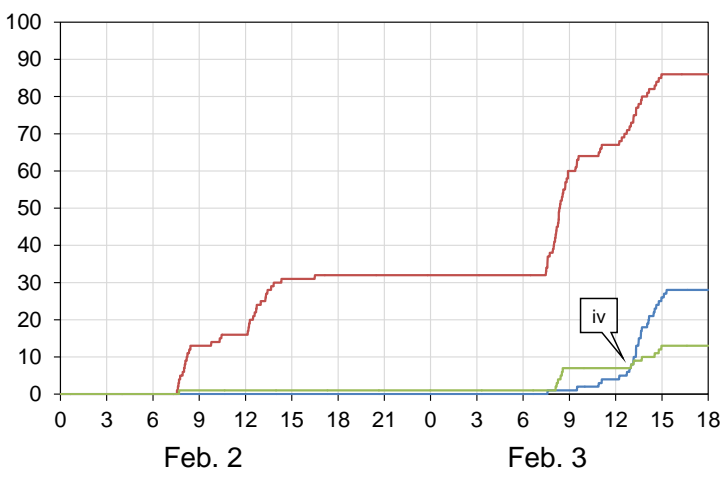

e) Dry

Figure 5. Cumulative classifications per user.

Figure 5d shows relatively close agreement between User 1 and User 3 for the "wet" classification, with User 2 lagging behind for most of the period. Callout iii indicates a moment just before noon on February 3 where User 3 ramps up the number of images classified in this category. Figure 5e, callout $i v$ shows the inverse of the dynamic, with User 1 having a greater increase in the "dry" classification before noon over User 3.

\subsection{Variations of Images Classified}

After classification, the median and deviation over the three users are computed for each image. Each category is assigned an index of 1 to 6 where each image is provided three indices, one for each user. The representative median classification for each image is then used for determining the 


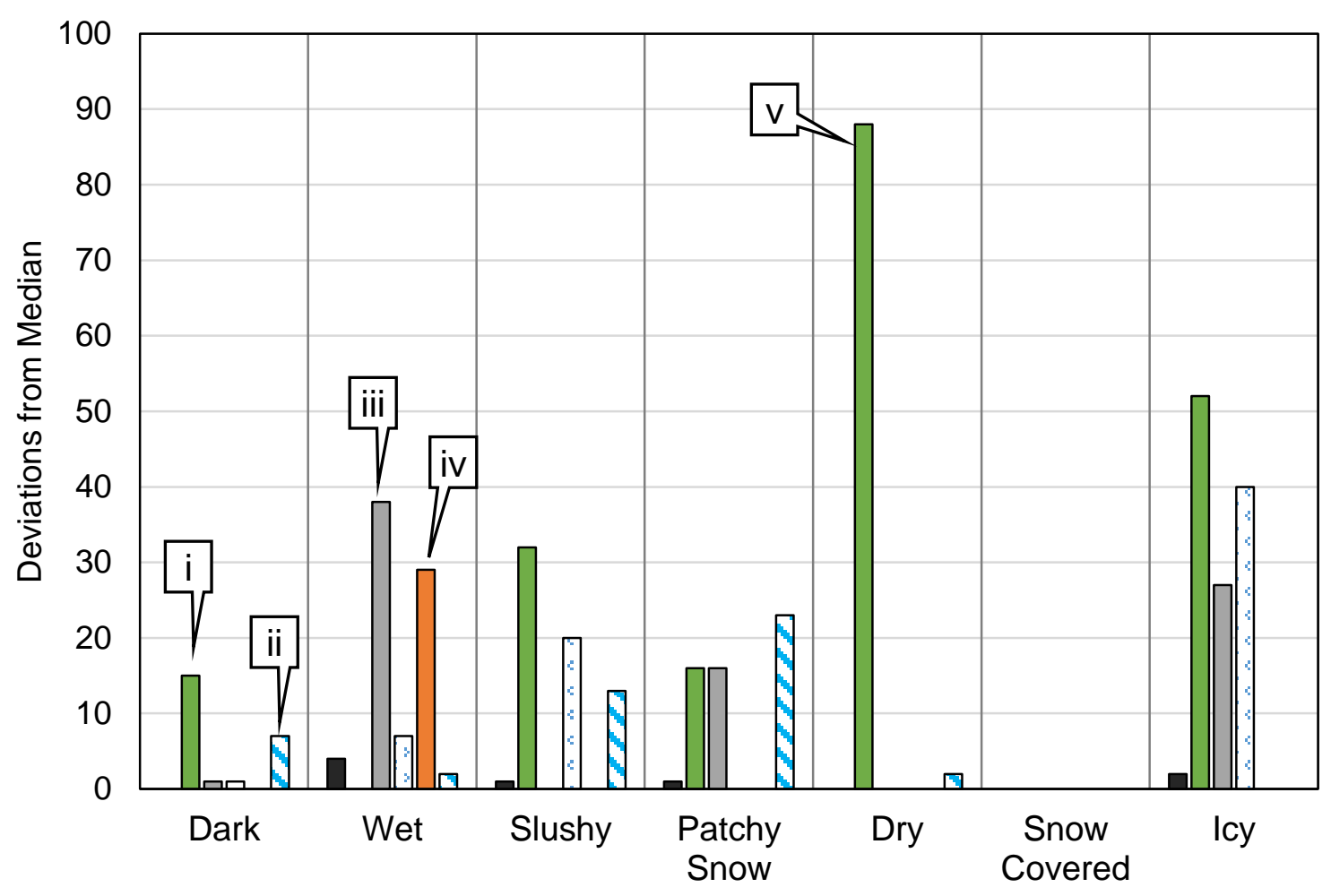

घDark $\square$ Wet $\square$ Slushy $\square$ Patchy Snow $\square$ Dry $\square$ lcy

Figure 6. Deviations from median classification.

deviation. Figure 6 illustrates the total number of images deviating from the median classification in each category. The categories on the $\mathrm{x}$-axis signify the median classification and each of the bars indicate the number of images in a different category deviating from the median classification. For instance, images classified as "dark" at the median has 15 images that are also classified as "wet" and seven images that are classified as "icy" (callout $i$ and $i i)$. This may be attributed to dim lighting during the dawn and dusk periods where users are less likely to be consistent. For images with the "wet" classification, 38 of those images are classified as "slushy" (callout iii) while 29 of those images are classified as "dry" (callout $i v$ ) and 7 images are classified as "patchy snow." For the "slushy" category, the images deviate to 32 "wet," 20 "patchy snow," and 13 "icy." The "patchy snow" classification has 16 images deviating to the "wet" and "slushy" category apiece, with 23 images deviating into the "icy" category. The "dry" category has the most number of images deviating to the "wet" category with 88 (callout $v$ ). Finally, the "icy" category has 52 images in the "wet," 27 images in the "slushy," and 40 images in the "patchy snow" categories. There are no images classified for "snow covered."

Out of the seven categorized, three are considered to be winter conditions: "patchy snow," "icy," and "slushy." "Icy" has the most number of deviations with 121 images classified into another category, followed by "slushy" with 66 images and "patchy snow" with 56 images. The "wet" category also shows substantial images classified as a winter condition. 


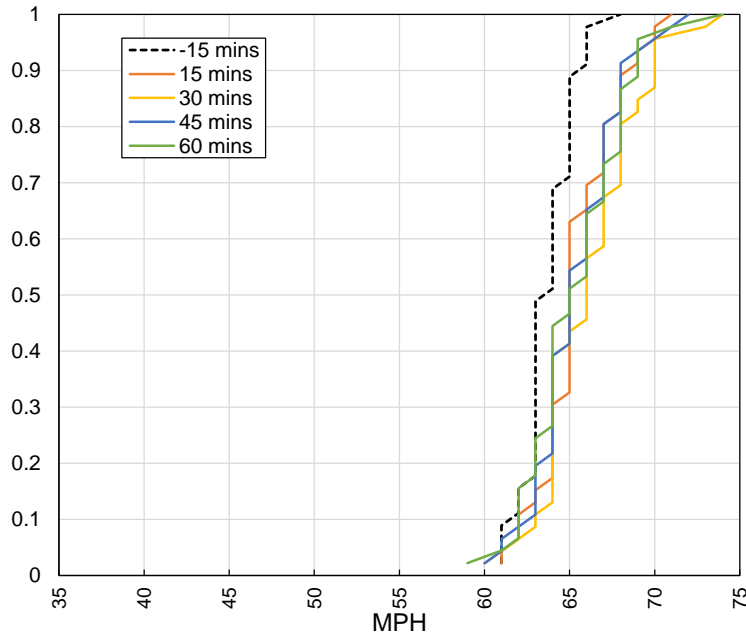

a) Patchy Snow

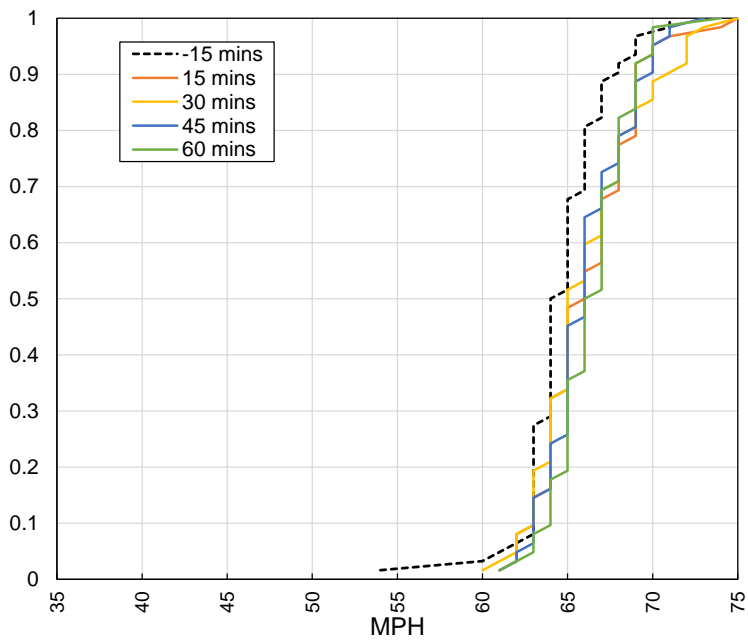

c) Slushy

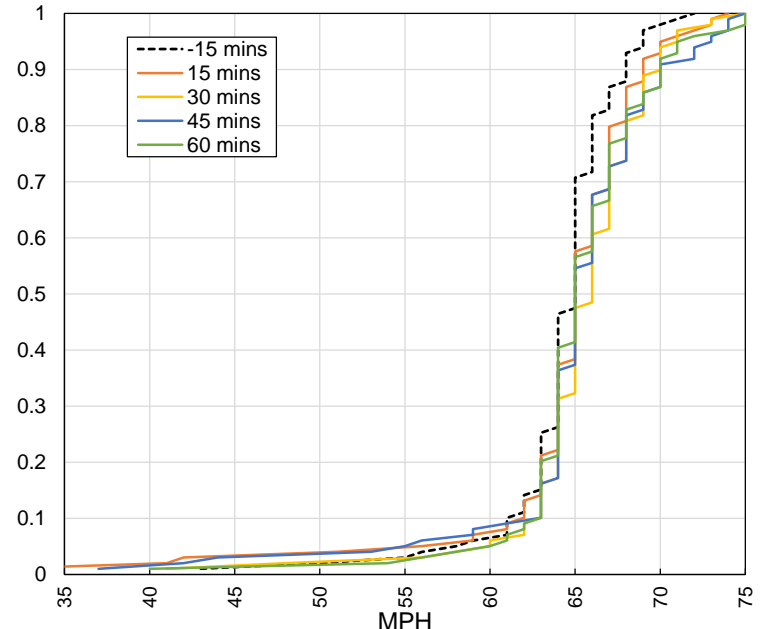

b) Icy

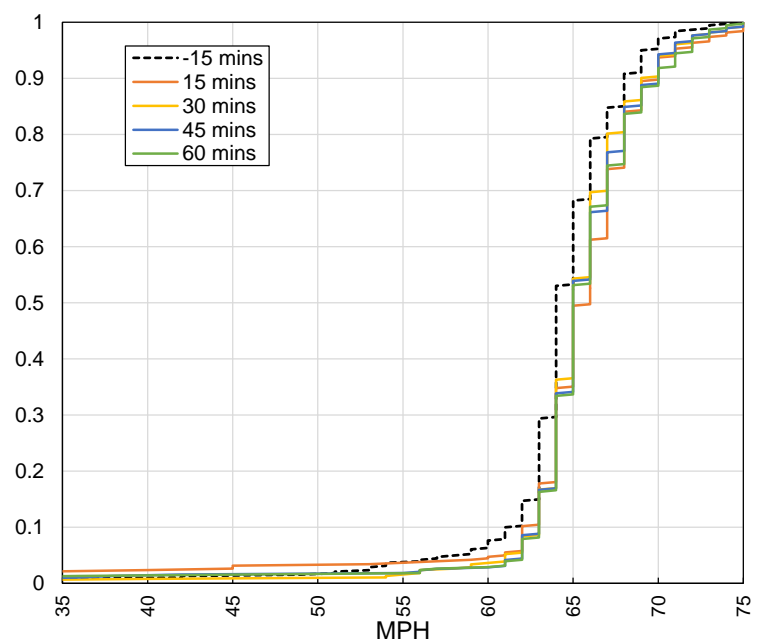

d) Wet

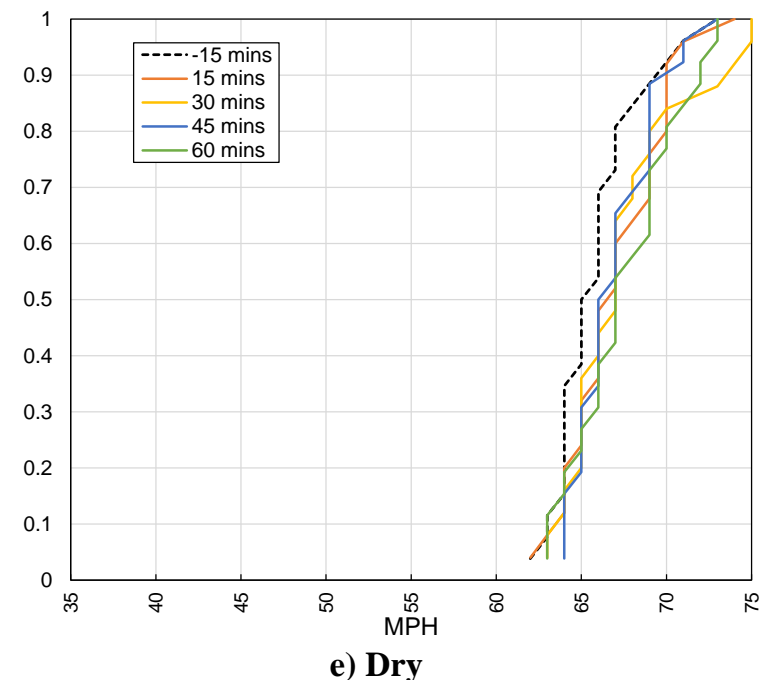

Figure 7. Speed distribution of segments before and after snow plow image. 


\subsection{Speed Distributions}

Connected vehicle speed data is used to assess traffic speeds on corresponding segments of roadway, in the direction of travel of the snow plow, in a two-hour window around the time of when each of the images was taken. Figure $7 \mathrm{a}$-e each show a series of cumulative distribution curves for speeds at predefined number of minutes before and after the image time. Each figure illustrates speeds related to images classified as "patchy snow," "icy," "slushy," "wet," and "dry" at 15 minutes before (dashed line) and 15, 30, 45, and 60 minutes after the time the image was taken. Figure 7a shows the speeds for "patchy snow" conditions before and after the plow were relatively similar for the slowest $25 \%$ of samples. From $25 \%$ to $50 \%$ of the samples, the speeds start to diverge where the after-plow conditions are 1 to $2 \mathrm{MPH}$ faster than speeds reported 15 minutes before the plow. For the top $10 \%$ of the quickest speeds in each time interval, there is up to a $6 \mathrm{MPH}$ improvement in the after time period, most substantially at 30 minutes after the plow has gone through.

Figure 7b shows less improvement between the before and after speeds for images that were classified as "icy." Moreover, this category of curves show much longer tails than the "patchy snow" classification. This indicates there is a wider distribution of speeds - as low as $31 \mathrm{MPH}$ fifteen minutes after the plow - for images that were classified as "icy." Figure 7c shows the distribution of speeds for the "slushy" classification. In this category, the speeds are not as widely distributed and there is a slightly greater improvement in speeds after the plow for the fastest $30 \%$ of samples. The slowest speeds for the "wet" classification in Figure 7d are the lowest among all classifications, at between $55 \mathrm{MPH}$ and $16 \mathrm{MPH}$ for the bottom 15\% slowest reported speeds for all reporting times before and after the plow. Finally, Figure 7e shows, as expected, the quickest speeds among all categories, between $62 \mathrm{MPH}$ and $75 \mathrm{MPH}$ for dry conditions. There is also a slight improvement for speeds reported after the plow has gone through the segment of $3 \mathrm{MPH}$ at the $80 \%$. This suggests that snow plows can further improve traffic speeds even as conditions were favorable to begin with.

\section{RESULTS}

\subsection{Impact Time of Plows}

Further investigation into the speed data is made to determine the time windows for which the plow has the largest impact on improving speeds. All possible speed distribution curves for locations classified as "patchy snow" are generated by minute for 60 minutes before and 60 minutes after the plow. The non-parametric Kolmogorov-Smirnov (KS) test is then applied to compare all combinations of the curves to generate 3,600 before-and-after scenarios. For each combination, the D-statistic, or the maximum vertical distance between a pair of speed distributions, is computed. The $\mathrm{D}$-statistic is then related to a D-critical value, the results of which are used to compute the statistical goodness-of-fit (10).

Figure 8 shows a heat map of P-values computed using the method over all combinations of minutes before and after the plow. The vertical axis signifies the number of minutes before the plow has gone through, and the horizontal axis signifies the number of minutes after the plow, whereas each cell signifies the P-value of the maximum vertical difference between the before and after speed distribution curves. The value of 1.00 is used for values of $\mathrm{P}$ greater than $\overline{999}$. For this data set we focus on the comparisons that produce the least goodness-of-fit. The cells are colored yellow at $\mathrm{P}=.95$ and blue as $\mathrm{P}$ approaches 0 . 
From the heat map, the time period for which a sample appears to consistently have some statistical significance appears to be from 17 to 12 minutes before the plow goes through. Sampled speeds appear to be most significantly different 25 to 35 minutes after the plow. The comparison of speeds 15 minutes before the plow and 28 minutes after the plow has the lowest P-value of 0.08 at callout $i$, which suggests that during this interval the plows have the greatest impact to road conditions, data latency notwithstanding. Other significant sample periods are 48 to 51 minutes after the plow and 54 minutes to 56 minutes after the plow.

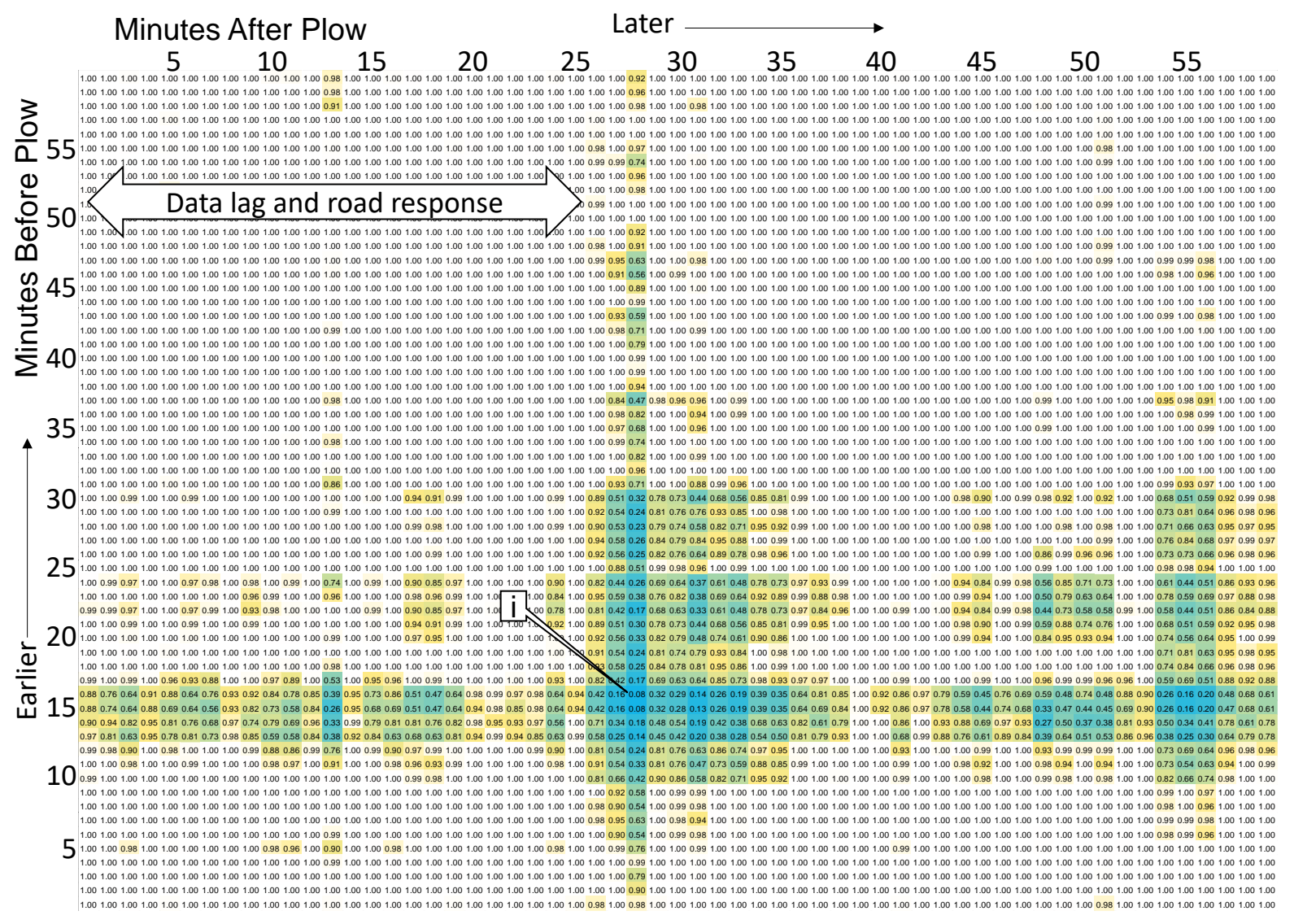

Figure 8. Heat map of KS-test P-values comparing speed distributions minutes before and after plow for images identified as "patchy snow."

\subsection{Time Series}

Time-series charts of the interquartile range (IQR) and minimum and maximum values of speed distributions are plotted Figure 9. Each box-and-whisker plot shows the distribution of speeds in each minute from 60 minutes before the plow to 60 minutes after the plow, for the winter categories of "patchy snow," "icy," and "slushy." Figure 9a shows the distribution of speeds for a "patchy snow" category. At callout $i$, the speeds range between $55 \mathrm{MPH}$ and $75 \mathrm{MPH}$ at 45 minutes before the plow goes through. Callout $i$ shows an instant 12 minutes before the plow where the highest speed drops to $69 \mathrm{MPH}$ and the lowest speed drops to $53 \mathrm{MPH}$. After the plow goes through, the top speeds improve back to $75 \mathrm{MPH}$ at callout iii.

Figure $9 \mathrm{~b}$ shows the time series of speed distributions for the "icy" classification. Callout iv shows some of the lowest minimum speeds before the plow at $27 \mathrm{MPH}$ compared to after the plow 
at $36 \mathrm{MPH}$ (callout $v$ ). Figure 9c shows that the greatest improvements in speed distributions are for the road conditions classified as "slushy." Callout $v i$ shows minimum speeds falling to 41 MPH before the plow and improves to a minimum of $55 \mathrm{MPH}$ to above $60 \mathrm{MPH}$ after the plow. These

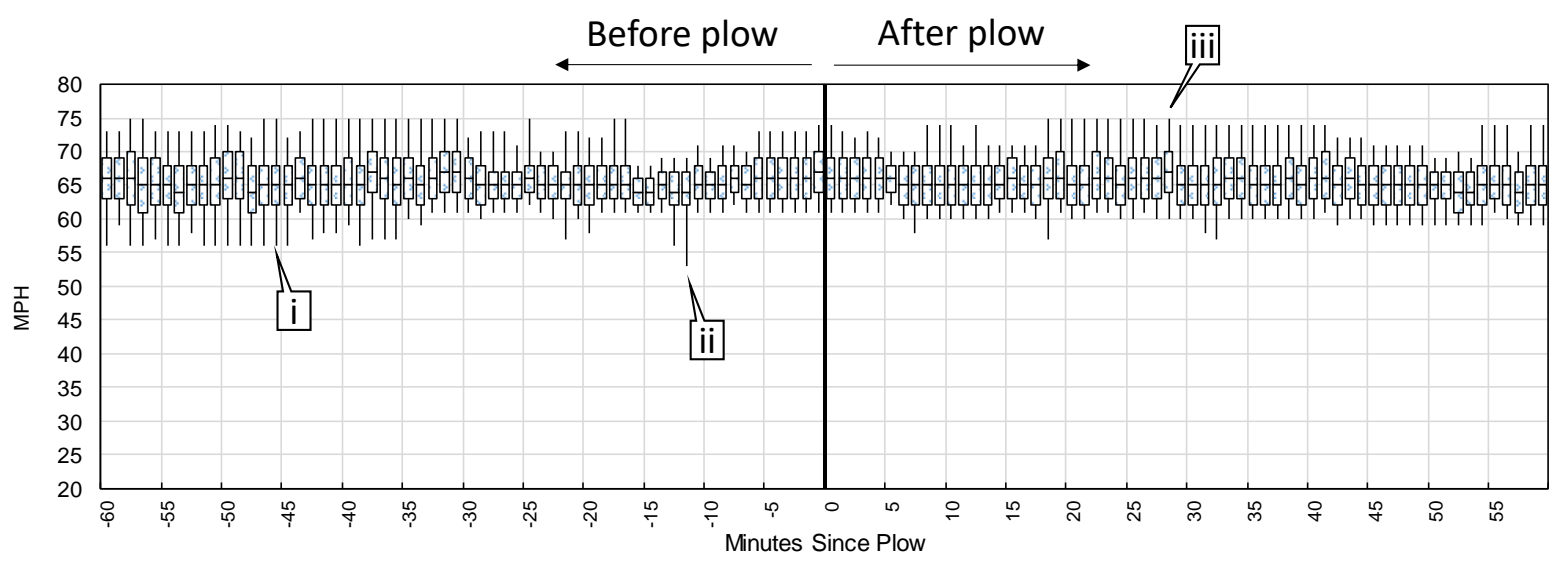

a) Patchy snow.

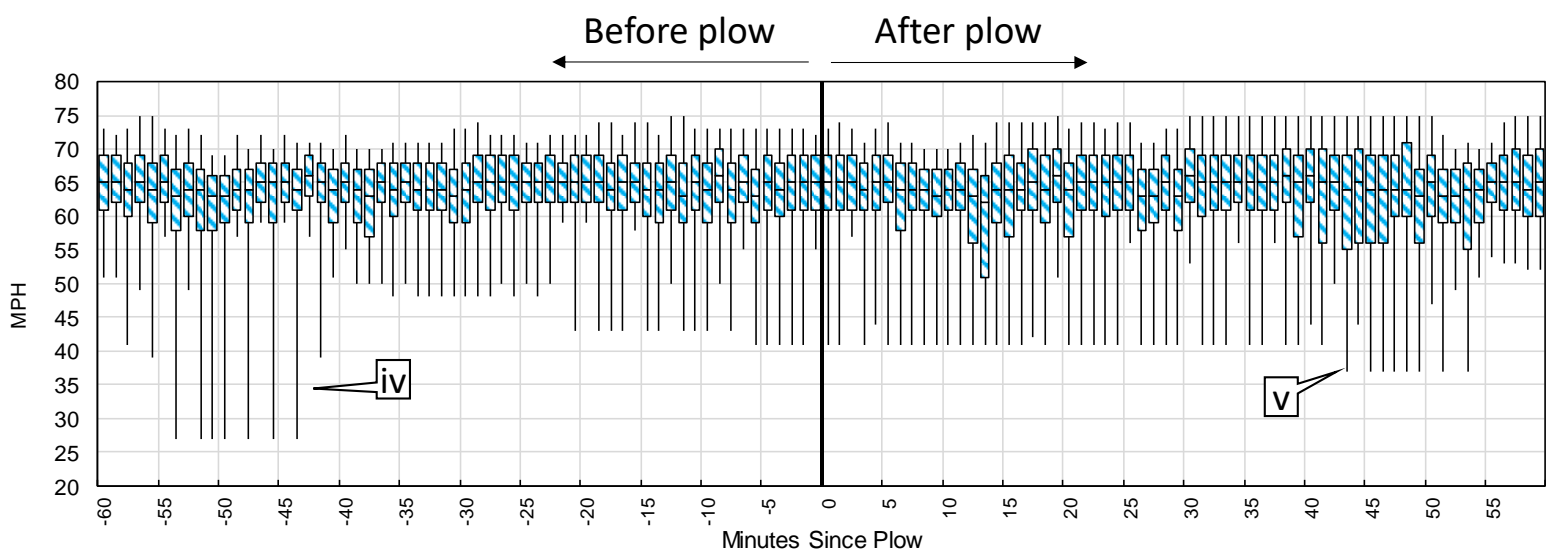

b) Icy.

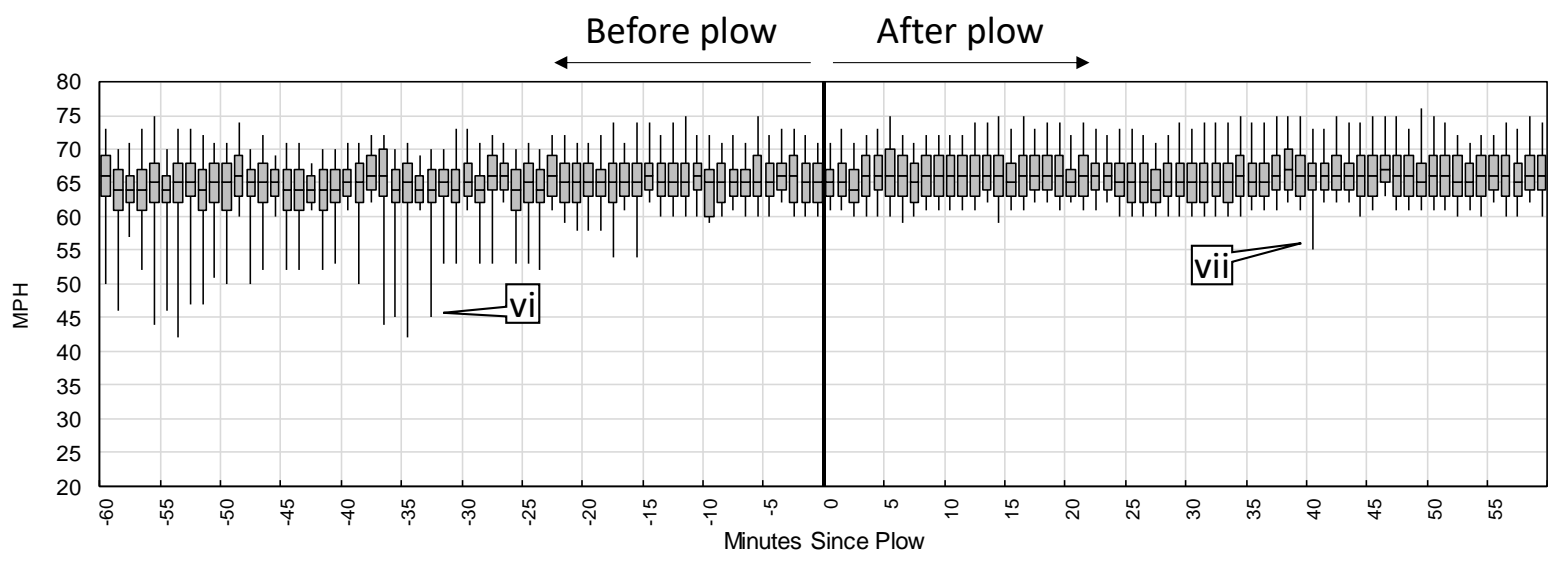

c) Slushy.

Figure 9. Distribution of speeds by winter condition categorized, before and after plow.

results suggests that while the plows do not significantly improve traffic speeds during wintery conditions for the largest population of samples, the minimum outliers, those which often may be indicators of dangerous driving conditions, are improved. 


\section{CONCLUSIONS}

In this study, 1,119 images taken from Iowa DOT AVL-equipped snow plows were examined for a storm on February 2-3, 2016. The images were classified by three users into one of seven categories that describe the road condition in each image. The classification of images saw the greatest deviation when road conditions were deemed "wet," "dry," or "icy," suggesting some variation in the interpretation of these categories by the users. Connected vehicle speed data for a segment was retrieved at the GPS location of each image, for a two-hour window around when the image was taken, to identify any potential impacts the plows made. The "patchy snow" category saw the greatest improvement after a snow plow went through, with a $6 \mathrm{MPH}$ improvement for the top $10 \%$ of speeds about 30 minutes after the plow has gone through.

A KS-test was performed to determine which comparisons were most significant between minutes before and after the plow. It was found that speed data taken between 17 to 12 minutes before the plow had the greatest maximum distance improvement on the speed distributions 25 to 35 minutes after the plow had gone through.

Finally, a two-hour time-series of speed distributions were analyzed for the winter categories. It was determined that while the plows did not have significant impact on IQR speeds, much of the minimum speeds recorded in the before scenarios were improved. Future opportunities from this study includes exploring which options are the best to include for a crowd-source type of weather assessment application, and optimal plow truck deployment frequencies based on the time-ofimpact of the greatest speed improvements.

\section{GLOSSARY}

Automatic Vehicle Location (AVL) - technology or devices that record the spatial location of a vehicle and supplemental data.

Comma-Separated Variable (CSV) file - an electronic file that stores tabular data separated by commas in plain text format.

Connected vehicle - a vehicle with internet or other network connectivity, and has the ability to share information from onboard instrumentation external to the vehicle.

Cumulative frequency diagram - a diagram that illustrates the running total of frequencies in order of value for a data set.

Geographic Information Systems (GIS) - electronic systems that store, manipulate, and display data relating to the earth's surface.

Intelligent Transportation Systems (ITS) - electronic systems that combine the use of different technologies that facilitate the monitoring, management and operation of various modes of transportation.

Interquartile Range $(I Q R)$ - the difference between the $75^{\text {th }}$ and the $25^{\text {th }}$ percentile of a data set as measure of variability. 
Kolmogorov-Smirnov $(K S)$ test - a statistical goodness-of-fit test that compares two empirical distribution functions using maximum distance.

Road Weather Information System (RWIS) - a system of weather data stations, communication technologies, and central systems to collect and disseminate weather field data.

\section{ACKNOWLEDGMENTS}

This work was supported in part by Iowa DOT and the Joint Transportation Research Program administered by the Indiana Department of Transportation and Purdue University. The connected vehicle speed data was provided by INRIX, Inc. The contents of this paper reflect the views of the authors, who are responsible for the facts and the accuracy of the data presented herein, and do not necessarily reflect the official views or policies of the sponsoring organizations. These contents do not constitute a standard, specification, or regulation.

\section{REFERENCES}

1. Snow and Ice. Federal Highway Administration. http://www.ops.fhwa.dot.gov/weather/weather_events/snow ice.htm, (accessed 07.31.16).

2. Khattak, A. and K. Knapp. Interstate Highway Crash Injuries during Winter Snow and Non-Snow Events. Transportation Research Board (TRB) Annual Meeting Conference Proceedings, 2001.

3. Wael M., ElDessouki, John N. Ivan, Emmanouil N. Anagnostou, Adel W. Sadek, Chen Zhang. Using Relative Risk Analysis to Improve Connecticut Freeway Traffic Safety under Adverse Weather Conditions. Final Report US DOT Region 1 UTC Project UCNR 14-5. 2004.

4. Hanbali, R. and D. Kuemmel. Traffic Volume Reductions due to Winter Storm Conditions. Transportation Research Record No. 1387. 1992.

5. Pisano, P., Goodwin, L., Rossetti, M. Highway Crashes in Adverse Road Adverse Weather Conditions. The 88th Annual Meeting American Meteorological Society, New Orleans, 2008.

6. Eisenberg, D. and K. Warner. Effects of Snowfalls on Motor Vehicle Collisions, Injuries, and Fatalities. University of California at Berkeley \& University of Michigan at Ann Arbor, American Journal of Public Health, Vol. 95, No. 1, 2005.

7. Maze, T. and Z. Hans. Crash Analysis to Improve Winter Weather Traffic Safety, TRB Annual Meeting. 2007.

8. Road Weather Forecast. Utah Department of Transportation. http://udottraffic.utah.gov/RoadWeatherForecast.aspx, (accessed 07.31.16).

9. FHWA. Iowa's Real-time data Integration of Snowplow Information and Roadway Safety Strategies for Using GIS to Advance Highway Case Study. FHWA-SA-16-030. 2016.

10. Day, C.M., D.M. Bullock. Calibration of Platoon Dispersion Model with High-Resolution Signal Event Data. Transportation Research Record, no. 2311. p. 16-28, 2012. 